\title{
Single-Trial Variability in Event-Related BOLD Signals
}

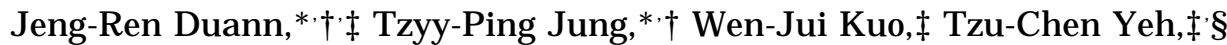

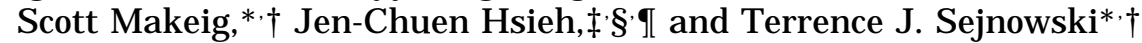

\begin{abstract}
*Computational Neurobiology Laboratory, The Salk Institute for Biological Studies, La J olla, California 92037; †Institute for Neural Computation, University of California San Diego, La J olla, California; łIntegrated Brain Research Unit, Department of Medical Research and Education, Taipei Veterans General Hospital, Taipei, Taiwan; and §Departments of Anesthesiology,

Psychiatry, and Radiology, Faculty of Medicine, School of Medicine, II nstitute of Neuroscience, School of Life Science, National Yang-Ming University, Taipei, Taiwan
\end{abstract}

Received J uly 26, 2001

Most current analysis methods for fMRI data assumea priori knowledge of the time course of the hemodynamic response (HR) to experimental stimuli or events in brain areas of interest. In addition, they typically assume homogeneity of both the HR and the non-HR "noise" signals, both across brain regions and across similar experimental events. When HRs vary unpredictably, from area to area or from trial to trial, an alternative approach is needed. Here, we use Infomax independent component analysis (ICA) to detect and visualize variations in single-trial HRs in event-related fMRI data. Six subjects participated in four fMRI sessions each in which ten bursts of 8-Hz flickering-checkerboard stimulation were presented for 0.5-s (short) or 3-s (long) durations at 30-s intervals. Five axial slices were acquired by a Bruker 3-T magnetic resonance imager at interscan intervals of $500 \mathrm{~ms}$ (TR). ICA decomposition of the resulting blood oxygenation level-dependent (BOLD) data from each session produced an independent component active in primary visual cortex (V1) and, in several sessions, another active in medial temporal cortex (MTN5). Visualizing sets of BOLD response epochs with novel BOLD-image plots demonstrated that component HRs varied substantially and often systematically across trials as well as across sessions, subjects, and brain areas. Contrary to expectation, in four of the six subjects the V1 component HR contained two positive peaks in response to short-stimulus bursts, while components with nearly identical regions of activity in long-stimulus sessions from the same subjects were associated with singlepeaked HRs. Thus, ICA combined with BOLD-image visualization can reveal dramatic and unforeseen $H R$ variations not apparent to researchers analyzing their data with event-related response averaging and fixed HR templates. 2002 Elsevier Science (USA)

Key Words: event-related fMRI; BOLD signal; hemodynamic response; independent component analysis; ICA; single-trial variability.

\section{INTRODUCTION}

Although functional magnetic resonance imaging (fMRI) of blood oxygen level-dependent (BOLD) contrast allows localization of dynamic brain processes during a wide range of psychological tasks (Ogawa et al., 1992; Kwong et al., 1992; Bandettini et al., 1993, 1997), BOLD data are complex mixtures of signals resulting from brain hemodynamics as well as from respiratory, movement-related, temperature-drift, machine-noise, and other processes. A number of methods have been proposed for analyzing BOLD data. These can be categorized as being either hypothesis-driven and model-based or else exploratory and data-driven (Friston, 1995, 1997). Model-based methods include analysis of variance (ANOVA) (Friston, 1995) and correlational methods (Bandettini et al., 1993). Datadriven methods include principal component analysis (PCA) (e.g. Moeller and Strother, 1991) and independent component analysis (ICA) (MCKeown et al., 1998a,b; McKeown and Sejnowski, 1998; McKeown, 2000; Arfanakis et al., 2000; Moritz et al., 2000; Calhoun et al., 2001).

Model-based methods usually assume that the form, though not the amplitudes, of the time courses of the different processes that sum to create the observed BOLD signals can be reliably estimated prior to analysis. Typically, the time course of stimulus presentation or task variation is convolved with a gamma, Poisson, or Gaussian response kernel, or else a combination of Fourier series are used to generate one or more expected hemodynamic response (HR) functions (Rajapakse et al., 1998; Kruggel and von Cramon, 1999a,b). The actual time courses of every voxel or smoothed voxel regions are then compared to the selected template(s) using a general linear model (GLM), fit by maximum likelihood or Bayesian statistics. Results are used to identify regions whose time courses are significantly correlated to the modeled behavior of interest, and to determine the magnitudes of their 
model-related activation (J osephs et al., 1997; Zarahn et al., 1997; Friston et al., 1998; Rowe, 2001). Genoveses et al. (1997) modeled observed BOLD signals by the sum of baseline, drift, activation, and noise components and then applied hypothesized template functions to the modeled activation component. However, all these methods assume that the HR time course is constant across stimulus or task events and, often, across brain areas, stimulus parameters, sessions, and subjects.

Hypothesis-driven methods may be problematic, however, when the HR time courses of interest are not stable and/or cannot be modeled with certainty prior to the analysis. For instance, recorded fMRI signals consist of changes in oxygenated hemoglobin concentration both in the capillary bed of each local cortical area and in its venous drainage (Gjedde, 1997). Since the drainage compartment may be located some distance from the capillary compartment, it may have a somewhat different BOLD time course (Frahm et al., 1994; Lee et al., 1995; Kansaku et al., 1998) making it difficult to construct single accurate a priori HR template. Variations in HR peak latency of up to $4 \mathrm{~s}$ in different brain regions are also reported, without current physiological explanation (Aguirre et al., 1998). In general, therefore, the basic model of the HR as a passive lowpass filter has limited validity for fMRI research.

Some research groups have begun to search for new, more flexible template-based methods for extracting accurate fMRI time courses or for data-driven methods for adjusting a priori HR models to the data. Williams et al. (2000) used orienting responses (event-related changes in heart rate, EEG desynchronization, eye movement, and skin conductance) to refine their HR model. Andino et al. (2000) utilized Renyi numbers of time/frequency representations on clusters of voxel time courses to measure signal complexity and taskrelated information content. Clare et al. (1999) demonstrated an ANOVA method data that calculates the ratio of the variance of the averaged data set to the variance of the unaveraged data for each voxel. This ratio, they reasoned, should be significantly higher for activated voxels. Their method assumes that the BOLD signals of "nonactivated" voxels are wholly unaffected by the time locking events. Although all the above methods may use less rigid assumptions about $\mathrm{HR}$ wave shape and timing than simple correlation methods, they still assume that the same HR is evoked in each trial and model trial-to-trial variability as statistical noise.

PCA, the best-known data-driven algorithm, is often employed to reduce the dimensionality of fMRI data. However, individual PCA components are necessarily both spatially and temporally uncorrelated, making them unlikely to represent functionally distinct brain systems. Rotation methods such as Varimax and Promax (Hendrickson and White, 1966; see Makeig et al.,
2000) might be used to relax the rigid orthogonality constraint, but their use for $\mathrm{fMRI}$ analysis has not been explored and the relevance of their underlying assumptions to $\mathrm{fMRI}$ signals may also be questioned.

McKeown and colleagues (1998a,b, 1998) reported a less artificially restrictive data-driven method, ICA, for blind source separation of many types of activity from fMRI data based on an information-maximization (I nfomax) algorithm of Bell and Sejnowski (1995). Components found by ICA applied to fMRI data are spatially maximally independent of one another, though their time courses may be correlated. This approach is compatible with the well-established principle of functional brain modularity (different parts of the brain do different things) and is capable of distinguishing signal sources with slightly differing time courses.

ICA attempts to decompose the entire fMRI data set into component activities associated with fixed spatial distributions. Variability in the data is modeled as a sum of deterministic processes with maximally independent spatial distributions. McKeown et al. (1998b) showed that ICA can identify and distinguish activity in brain regions having consistently task-related HRS from those whose HRs are only transiently task-related. ICA can also isolate other metabolic and artifactual processes, such as radiofrequency (RF) noise, subject movements and arterial pulsations in fMRI data, allowing their removal prior to further analysis (J ung et al., 2001a,b). This paper demonstrates that ICA, applied to event-related fMRI paradigms, can reveal BOLD processes having novel and highly variable HRs, even in simple sensory response paradigms, allowing detailed examination of HR variations across trials, sessions, sites, and subjects.

\section{METHODS}

\section{Subjects}

Two male and four female subjects (ages $22 \pm 3$ years, mean $\pm S D$ ) were recruited from the academic environment of the National Yang-Ming University in Taipei, Taiwan. Each subject had normal or correctedto-normal vision. Written informed consent was obtained from each subject prior to the experiment.

\section{Experimental Protocol}

An overview of the experimental design is shown in Fig. 1. An 8-Hz flickering visual checkerboard stimulus was used to trigger HRs. Each 5-min experimental session consisted of ten 30-sec epochs beginning with a burst of visual stimulation. The 30-s stimulus onset asynchrony (SOA) was chosen to minimize the possibility of HR overlap (Buckner, 1998; Buckner et al., 1998a,b; Dale, 1999). In periods between stimulus bursts, subjects were requested to fix their eyes on a 


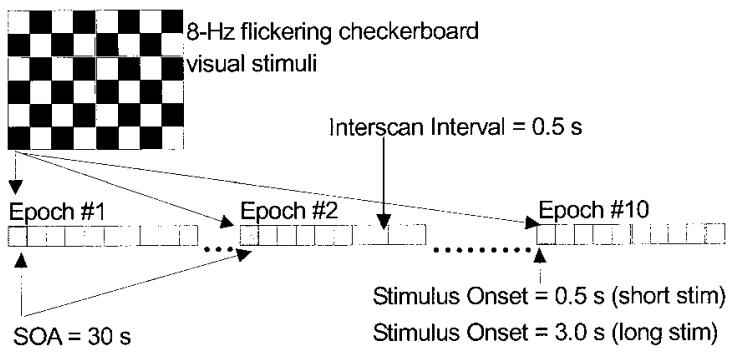

FIG. 1. Experimental paradigm. Visual 8-Hz flickering-checkerboard stimuli were presented at the beginning of each 30-s epoch. Stimulus duration was $0.5 \mathrm{~s}$ in short-stimulus sessions and $3 \mathrm{~s}$ in long-stimulus sessions. Each session comprised ten 30-s epochs. Six subjects participated in two short-stimulus and two long-stimulus sessions.

red crosshair displayed in the center of visual field. Visual stimuli were presented through back projection from an LCD projector onto a white screen. Subjects lay on their backs in the bore of the scanner and viewed the screen through eyeglasses containing an angled mirror in front of each eye. In short-stimulus (SS) sessions, stimulus burst duration was $0.5 \mathrm{~s}$; in longstimulus (LS) sessions, burst durations were $3 \mathrm{~s}$. Each subject participated in two LS and two SS sessions whose order was counterbalanced over sessions and subjects.

\section{Image Acquisition}

fMR images were obtained by a 3-T Medspec 30/100 scanner (Bruker Medizintechnik GmbH, Ettlingen, Germany) at the Integrated Brain Research Unit (IBRU) of Taipei-Veterans General Hospital, Taipei, Taiwan. Five axial slices were acquired using an echoplanar imaging (EPI) protocol $(\mathrm{TR}=500 \mathrm{~ms} ; \mathrm{TE}=70$ $\mathrm{ms}$; flip angle $=90^{\circ}$; matrix $=64 \times 64 ; \mathrm{FOV}=250 \times$ $250 \mathrm{~mm}$; slice thickness $=5 \mathrm{~mm}$ with 2-mm gap). For better visualization of the active brain areas, $256 \times$ 256 T1-weighted images with the same slice position and field of view as the functional images were acquired at the end of the four experimental sessions.

\section{Data Preprocessing}

The obtained fMRI images were first subjected to a slice time-alignment process to minimize image intensity inhomogeneity arising from differences in slice image acquisition timing. In this process, data from individual slices were first interpolated with ideal lowpass interpolation in time. Then, the data were sampled at a single set of time points separated by the original 500-ms TR. If left uncorrected, the staggered acquisition times for the different slices might have introduced variability and timing bias into the recovered HRs (Aguirre et al., 1998). The timerealigned fMRI images were then stripped of off-brain voxels by threshol ding intensity histograms of the structural im- ages. This process reduced data size by more than $78 \%$ (to $4400 \pm 680$ within-brain voxels). Independent component analysis was then applied to BOLD-signal time series of within-brain voxels to separate the data into a sum of activity in maximally independent brain maps and find the associated BOLD-signal time courses.

\section{Independent Component Analysis}

In any analysis of fMRI data using a linear model, the time course of each brain or nonbrain voxel is considered to be the sum of a number of defined or undefined time courses expressed with differing strengths (and, possibly, polarities) across the space of voxels. Some linear models also assume that each voxel time course contains some level of independent and identically distributed noise, often modeled as Gaussian (Friston et al., 1995; Rowe, 2001). Sources of the constituent activities include ongoing and task-related hemodynamic changes, blood or central spinal fluid (CSF) flow patterns, and subject movements and machine artifacts. In linear decomposition theory, these activity sources are considered to represent different "component processes," each having a unique time course and spatial extent, that produce simultaneous changes in the fMRI signal at the individual signalspace voxels (McKeown et al., 1998a,b; McKeown and Sejnowski, 1998). In ICA, the component processes are assumed to sum to the measured signals, without prespecified noise terms, though some components may in fact include or represent one or more types of "noise" in the data.

Each fMRI component is represented by a spatially fixed three-dimensional "component map" (the weight for each voxel) and a single associated activity time course. ICA produces component maps that are maximally spatially independent. This means that the collection of voxel weights in each component map is maximally independent of the voxel weights for all the other component maps or, equivalently, that the map weights of any component contain minimal information about the weights of any other map. Note that for $\mathrm{fMRI}$ data, in which the number of time points is typically much smaller than the number of spatial voxels, spatial independence is assumed (McKeown et al., 1998a), whereas in other time series applications of ICA, such as el ectroencephalographic (EEG) decomposition, the desired components are assumed to have independent time courses (Makeig et al., 1996). The number of independent components of an fMRI data set separated by spatial ICA, therefore, is at most equal to the number of time points in the data. However, when ICA is applied to a lower-dimensional principal subspace of the data, the number of components is equal to the dimensions of the subspace.

At each acquisition time, $t$, the total measured signal at each voxel is considered to be the sum of the com- 
ponent activities, at time $t$, of each component time course weighted by the voxel's strength in the respective component maps (see McKeown et al., 1998b, for further discussion). Using I nfomax ICA, we can determine an unmixing matrix, $\mathrm{W}$, and spatially independent component activations, $\mathrm{M}$,

$$
M=W X
$$

where $X$ is the $T$ (times) by $V$ (voxels) matrix of $T$ BOLD signals, $W$ is $C$ (components) by $T$ (times), and $M$ is $C$ by $V$. When $W$ is invertible (as is al ways the case when $X$ has rank $T$ ), we may write

$$
\mathrm{X}=\mathrm{W}^{-1} \mathrm{M}
$$

A useful interpretation of Eq. (2) is that the columns of $\mathrm{W}^{-1}$ represent the time course of each component map in the data. That is, each component's contribution to the BOLD signal at each voxel is the product of the component time course value and the voxel's weight in the component map. When $\mathrm{W}$ is not full rank (e.g., in principal subspace decomposition), the MoorePenrose pseudo-inverse of $\mathrm{W}$ is used in (2).

Although extended Infomax (Girolami, 1998; Lee et al., 1999) is capable of finding components whose maps have nonnegligible weights in a large portion of the voxels, reports to date have found that independent BOLD components have relatively small regions of nonnegligible weight values. This tendency is reflected as a positive kurtosis of the component map weight distribution allowing making unnecessary the use of extended I nfomax (McKeown et al., 1998b). When some maps (i.e., rows) of $M$ are replaced with zeros, the resulting $X_{0}=W^{-1} M_{0}^{\prime}$ is the back-projection into the original BOLD signal voxel space of the remaining (nonzero) component(s).

The ICA unmixing matrix for each $\mathrm{fMRI}$ session was computed using a binary version of the runica() routine (available online in MATLAB and binary form) of Makeig et al . (1998). PCA preprocessing was applied to reduce the dimension of training data set from 600 (the number of time points) to 50 (i.e., ICA was applied to the 50-dimensional principal subspace of the data). The PCA reduction preserved $96 \%$ of the original signal variance. The initial value of the runica() learning rate (size of step to reduce weight in each iteration) was 0.0001; block size (number of data points chosen randomly in each iteration) was 34. Default values were used for other parameters. The complete procedure (including preprocessing) required $30 \mathrm{~min}$ on a 500-MHz Pentium 4 machine.

Hereafter the ICA training converged, (i.e., the unmixing matrix was determined within the preset tolerance), the spatially independent component maps were derived by $\mathrm{Eq}$. (1). Each component map (row of $\mathrm{M}$ ) was then normalized by subtracting its mean from each voxel and dividing by the standard deviation of the map weights. The voxels with $z$ values above a heuristic threshold (|z| > 2) were considered to comprise the component map or region of activity (ROA). We used quadratic normalization for heuristic purposes only, without assuming precise probabilistic implications, as the map weight distributions returned by ICA were al ways super-Gaussian. The threshold we chose (| z| > 2) appeared to give good results. Better motivated selection of threshold criteria is an important subject for future research.

To study the relationship between the component time courses derived by ICA and the time courses of relevant voxels in the raw data, we derived a positive ROA-mean time course for each component of interest by computing the unweighted average of the raw BOLD time courses of the positive-weighted $(z>2)$ voxels in the component ROA.

\section{Talairach Normalization}

To visualize the ROA of each component within the high-resolution structural image, and to compare ROAs across subjects, the structural images and component maps were normalized to the standard Talairach space using statistical parametric mapping (SPM) software from the SPM 99b package (Friston et al., 1995). Selected active component maps were then overlaid onto the structural images using the slice display() routine developed by M. Brett for SPM environment (see Fig. 2). The coordinates in Talairach space of active brain areas were obtained by locating the voxel with the largest $z$ value in each component ROA voxel cluster of interest. The Brodmann area(s) of the ROA cluster were determined from an online atlas (Lancaster et al., 2000). Because of the variability of HRs in different brain areas and subjects, we selected ICA components for further analysis based on their ROA rather than their time course. Components with positive ROAs located in Brodmann areas (BA) 17 and 19 could be selected automatically for further visualization and comparison.

\section{BOLD I mage Plots}

To display the time course of BOLD activity in a session, we used a new visualization tool we call the "BOLD-image" plot after a similar plotting method, the "ERP image," for event-related EEG data visualization (J ung et al., 2001a,b; Makeig et al., 2000). The time course of activation of each component was first converted to regional percent signal change (RPSC) by dividing the positive ROA-mean back-projected component time course by the positive ROA-mean BOLD signal level. For visual convenience, the 10 single-trial HRs were then smoothed with a two-trial wide moving 
TABLE 1

The Coordinates in Talairach Space of the Voxel with Maximal z Value in the V1 (BA17) Component Maps

\begin{tabular}{|c|c|c|c|c|c|c|}
\hline \multirow[b]{2}{*}{ Subject } & \multirow[b]{2}{*}{ Session } & \multicolumn{3}{|c|}{ Coordinates (L/R, mm) } & \multirow{2}{*}{$\begin{array}{c}\text { Significance }(L / R) \\
\text { Z score }\end{array}$} & \multirow{2}{*}{$\begin{array}{c}\text { Comp. inder } \\
(\mathrm{L} / \mathrm{R})\end{array}$} \\
\hline & & $x$ & $y$ & z & & \\
\hline \multirow[t]{3}{*}{$1^{\mathrm{a}}$} & SS1 (1) & $-18 / 16$ & $-78 /-80$ & $6 / 6$ & $6.10 / 4.04$ & $25 / 25$ \\
\hline & SS2 (2) & $-8 / 6$ & $-88 /-82$ & $4 / 12$ & $6.47 / 3.73$ & $25 / 25$ \\
\hline & LS (3) & $-4 / 12$ & $-90 /-82$ & $-6 / 6$ & $4.00 / 5.48$ & $43 / 43$ \\
\hline \multirow[t]{4}{*}{2} & SS1 (1) & $-4 /-$ & $-90 /-$ & $-4 /-$ & $4.36 /-$ & $16 /-$ \\
\hline & SS2 (4) & $-4 / 10$ & $-90 /-92$ & $-4 /-2$ & $5.71 / 4.87$ & $22 / 22$ \\
\hline & LS1 (2) & $-4 / 12$ & $-90 /-88$ & $-4 / 4$ & $6.87 / 4.33$ & $29 / 48$ \\
\hline & LS2 (3) & $-8 / 8$ & $-92 /-80$ & $4 / 12$ & $7.03 / 4.86$ & $49 / 18$ \\
\hline \multirow[t]{4}{*}{3} & SS1 (2) & $-10 / 8$ & $-88 /-80$ & $4 / 12$ & $3.58 / 4.52$ & $50 / 50$ \\
\hline & SS2 (3) & $-1-$ & $-1-$ & $-1-$ & $-1-$ & $-1-$ \\
\hline & LS1 (1) & $-6 / 14$ & $-102 /-94$ & $-2 / 4$ & $4.67 / 5.53$ & $9 / 9$ \\
\hline & LS2 (4) & $-22 / 8$ & $-94 /-76$ & $-8 / 12$ & $4.63 / 3.19$ & $21 / 8$ \\
\hline \multirow[t]{4}{*}{4} & SS1 (1) & $-2 / 8$ & $-82 /-94$ & $12 / 0$ & $3.87 / 4.56$ & $47 / 47$ \\
\hline & SS2 (4) & $-4 / 4$ & $-82 /-88$ & $12 / 6$ & $7.08 / 5.75$ & $36 / 36$ \\
\hline & LS1 (2) & $-4 / 8$ & $-92 /-98$ & $2 / 0$ & $5.28 / 7.64$ & $18 / 18$ \\
\hline & LS2 (3) & $-8 / 8$ & $-98 /-96$ & $0 / 0$ & $10.90 / 8.65$ & $36 / 36$ \\
\hline \multirow[t]{4}{*}{5} & SS1 (2) & $-12 / 2$ & $-88 /-88$ & $4 / 4$ & 7.19/8.74 & $42 / 42$ \\
\hline & SS2 (4) & $-14 / 12$ & $-94 /-90$ & $-12 /-10$ & $2.65 / 3.56$ & $10 / 23$ \\
\hline & LS1 (1) & $-2 / 2$ & $-86 /-88$ & $4 / 4$ & $6.12 / 9.12$ & $28 / 28$ \\
\hline & LS2 (3) & $-2 / 2$ & $-84 /-84$ & $4 / 4$ & $6.97 / 10.82$ & $45 / 45$ \\
\hline \multirow[t]{4}{*}{6} & SS1 (1) & $-14 / 4$ & $-78 /-88$ & $10 / 8$ & $5.72 / 3.34$ & $39 / 39$ \\
\hline & SS2 (4) & $-14 / 22$ & $-78 /-94$ & $10 /-8$ & $5.14 / 4.36$ & $31 / 24$ \\
\hline & LS1 (2) & $-14 / 24$ & $-78 /-96$ & $10 / 28$ & $5.14 / 4.74$ & $44 / 21$ \\
\hline & LS2 (3) & $-2 / 14$ & $-90 /-92$ & $0 / 0$ & $3.50 / 5.08$ & $46 / 46$ \\
\hline
\end{tabular}

Note For each subject, the structural and z-value images were normalized to Talairach space for labeling the active brain areas. The number in parentheses in the "Session" field indicates the session number (1st through 4th). L/R, left or right hemispheres. The coordinates were in $\mathrm{mm}$.

a Only three sessions acquired for Subject 1.

window applied separately, to each time point in the HR epoch. The smoothed component time courses in the 10 trials were then represented as a series of 10 horizontal bars variably colored according to the RPSC value at each time point. Finally, the 10 bars were stacked in trial order to form a BOLD image (Fig. 2b). For convenience, the average HR time course in the 10 trials was computed and drawn below the BOLD image. BOLD image-plots can effectively visualize the variability across trials of single voxel, region-of-interest (ROI) or component ROA time courses from eventrelated paradigms, providing clear visualization of response stability and variability across trials or sessions.

\section{Reproducibility within Subjects}

Within the same subject, the percent overlap ratio (POR) of the positive ROAs of similar components in two different sessions was derived as

$$
\begin{aligned}
P O R & =100 \\
\times N\left(R O A_{1} \cap R O A_{2}\right) & \sqrt{N\left(R O A_{1}\right) \times N\left(R O A_{2}\right)},
\end{aligned}
$$

where $N(S)$ is the number of elements in set $S$, and $\mathrm{ROA}_{1}$ and $\mathrm{ROA}_{2}$ are the sets of voxels in the positive ROAs of the two components or sessions. The $\cap$ operator measures the common voxels appear in both $\mathrm{ROA}_{1}$ and $\mathrm{ROA}_{2}$ and operator $\times$ gives the product of the number of voxels in $R O A_{1}$ and $R O A_{2}$. POR can be used to measure, for example, the percentage of active voxels in a component ROA from one session decomposition that are also in the ROA of a component from the another session decomposition. Presumably, if a brain region exhibits spatially coherent activity in different sessions, ICA should be able to find the same active component map in each session. Consequently, the overlap ratio can be used to estimate the reproducibility of an ICA component across sessions. 
a IC28 Region of Activity (ROA)
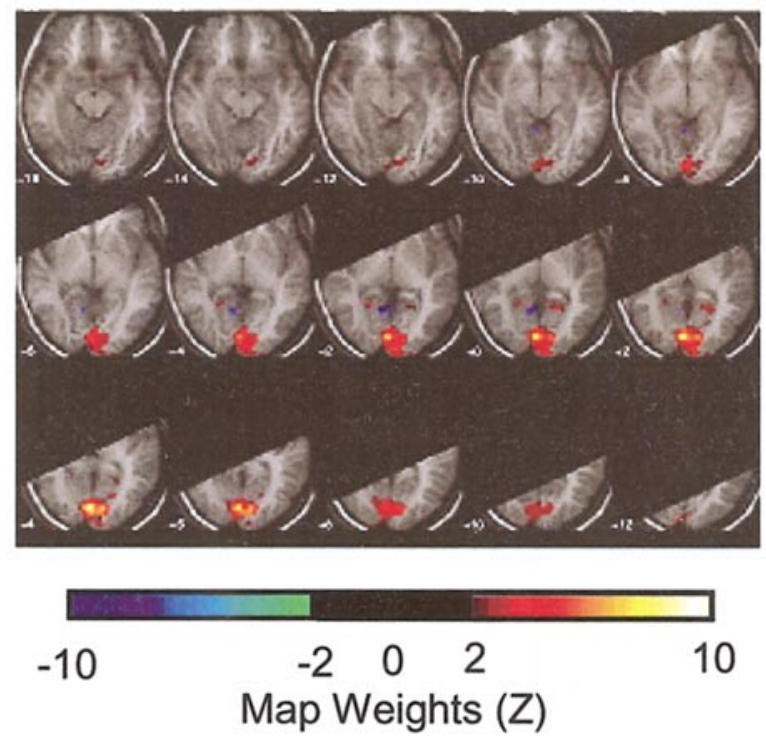

b IC28 BOLD Image Plot

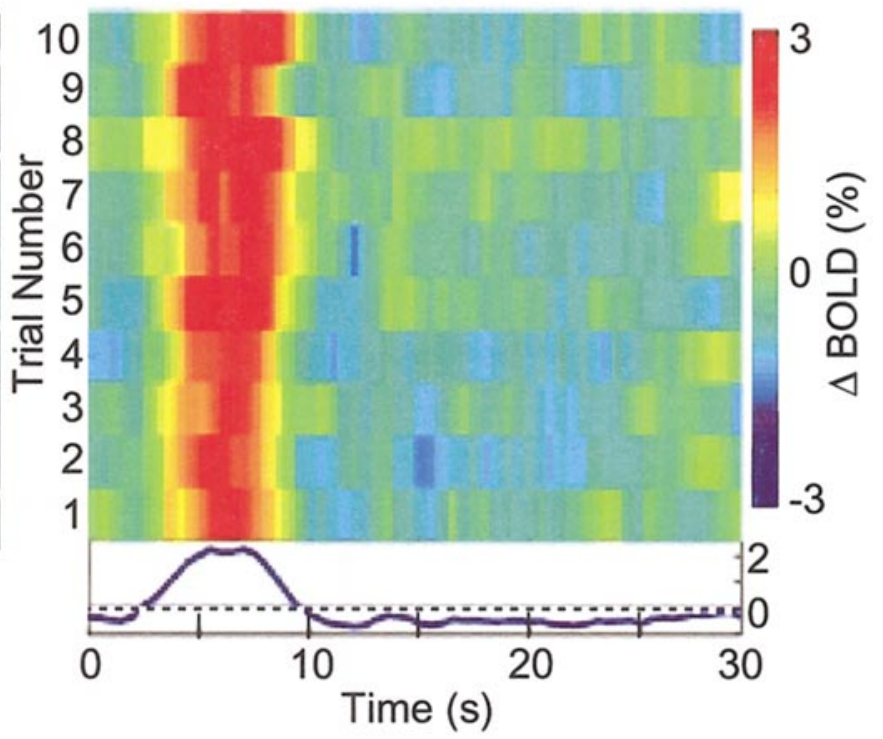

c IC28 ROA Decomposition

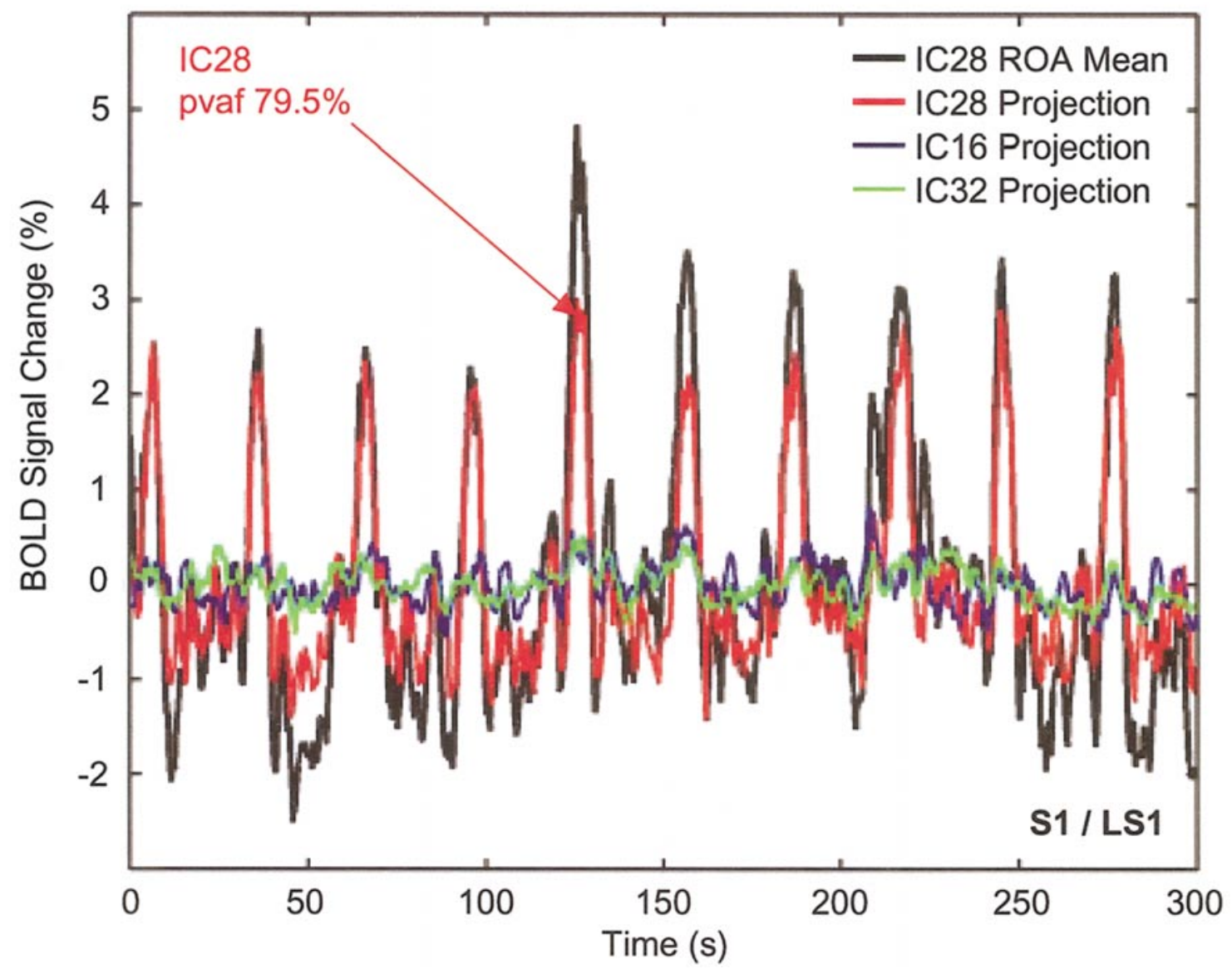

FIG. 2. BOLD image of an independent component active in V1. It shows: (a) the component region of activity (ROA) for component IC28 from the decomposition of a long-stimulus session from Subject 1 . Note the regions of positive (red) and negative (blue) activity. (b) A BOLD-image plot of the IC28 time course; and (c) the IC28 positive-ROA mean BOLD time course of voxels in the IC28 positive ROA (black trace), plus the mean IC28 time course (red trace) determined by back-projecting the ICA component to the positive ROA. 

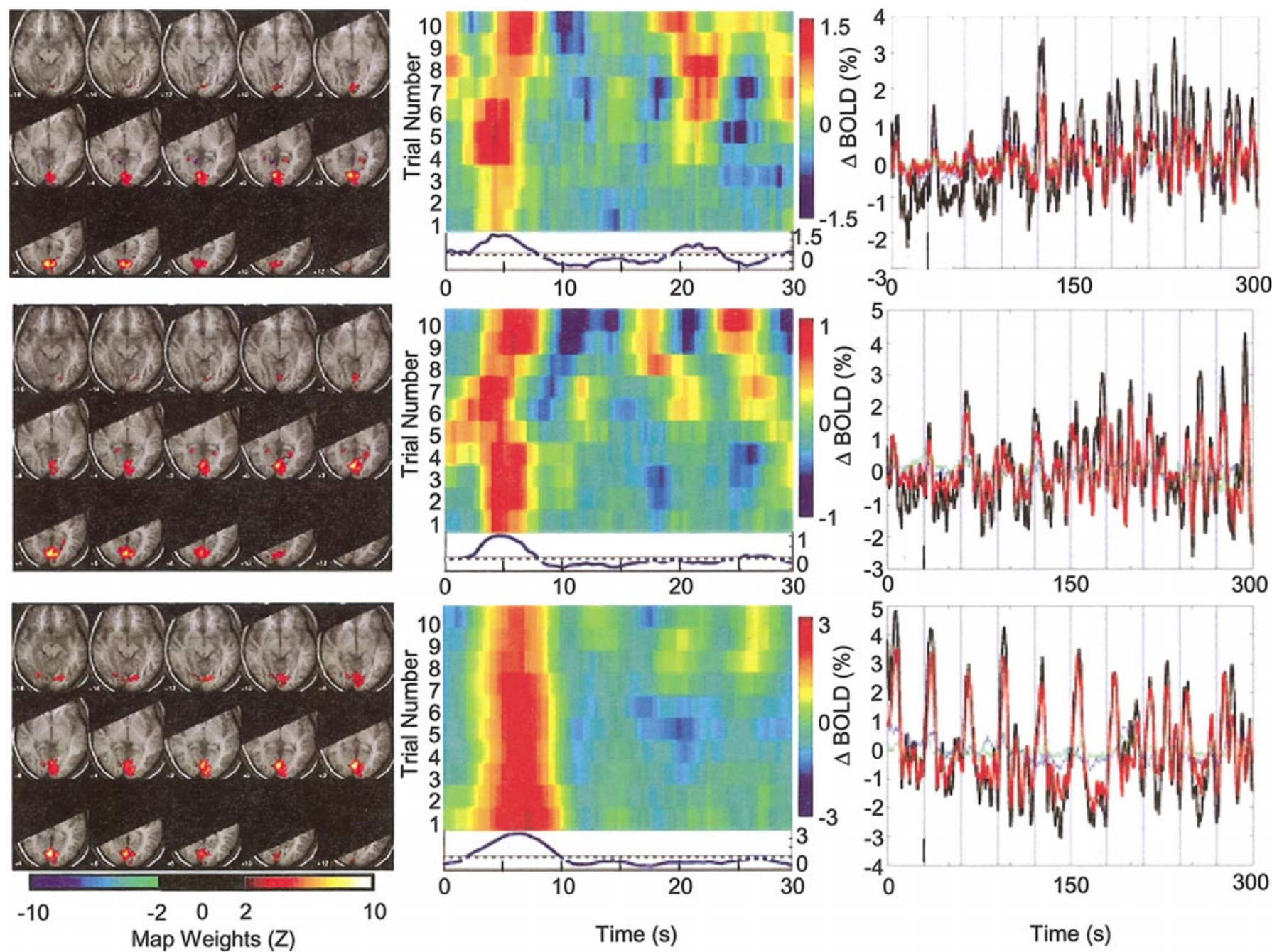

FIG. 3. Comparison of the V1 component time courses in the other three sessions from Subject 1 . The top row shows the result in the other long-stimulus session; the middle and bottom rows show results for the two short-stimulus sessions. The left column shows the region of activity (ROA) map superimposed on the MR structural image. The right column shows the component positive ROA mean time courses in the raw data (black trace) and ROA-mean time courses of the back-projected component (red trace), and the middle column shows BOLD-image plots of the mean time course of the component activity in the positive ROA.

To determine the salience of a selected component $\mathrm{k}$ in the raw data, its mean back-projected time course over the positive ROA voxels in the original data space $\left(X_{k \mid R O A}\right)$ was compared to the mean positive ROA time course in the raw data $\left(X_{R O A}\right)$ by computing the percentage of variance accounted for by the component $k$.

$$
\mathrm{PVA}_{\mathrm{k}}=100 \times\left(1-\frac{\operatorname{var}\left(\mathrm{X}_{\mathrm{ROA}}-\mathrm{X}_{\mathrm{kROA}}\right)}{\operatorname{var}\left(\mathrm{X}_{\mathrm{ROA}}\right)}\right) \text {. }
$$

\section{RESULTS}

For each session, components with positive ROAs concentrated in BA 17 and 19 were selected for further analysis. ICA returned one component in each session whose positive ROA was centered in BA17 (V1). Table
1 gives the coordinates, maximum $z$ value and component index of the components with ROAs concentrated in BA 17. Figure $2 a$ shows the component ROA and Fig. $2 b$ the component time course plotted as a BOLD image of the V1 component in the first long-stimulus session from Subject 1 (S1/LS1). Figure 2c plots the time courses of the ROA mean (black trace) obtained by averaging the time courses of the positive-weighted voxels within the IC28 ROA (Fig. 2a, red voxels) and the three components contributing to the ROA mean (colored traces). The red trace in Fig. 2c shows the ROA mean time course of the back-projected ROA-defining component (I C28). The component accounted for $79.5 \%$ of the variance in the whole data ROA mean. Other components also contributed to the ROA mean, but not strongly; the strongest are shown in Fig. 2c as blue and 
green traces. To form the BOLD-image plot (Fig. 2b), the time course of the defining component (red trace) was first segmented into successive 30-s trials. The 10 trials were then smoothed using a two-trial moving average, color coded, and stacked in trial order.

The left panel of Fig. 3 shows the V1 (BA 17) component ROAs for the other three sessions from Subject 1 . Within subjects, the active maps of the V1 components were nearly identical across sessions, presumably because the active area in primary visual cortex was the same in each session. Between long-stimulus sessions, the POR overlap ratio was $87.2 \%$; between long- and short-stimulus sessions, $82.1 \%$. However, the time courses of hemodynamic activity of these components (shown in the Fig. 3 BOLD-image plots) differed markedly across trials, sessions, and stimulus types. In later trials of both short-stimulus sessions (SS1, SS2), the V1 component time course included two major peaks separated by $\sim 17 \mathrm{~s}$, the latency of the second peak increasing systematically across trials. The HRs of the corresponding V1 components in the long-stimulus sessions, however, contained only one peak which had larger amplitude and longer duration than the initial peak of the short-stimulus session HRs.

The right column of Fig. 3 shows the ROA-mean time courses (black traces) together with the ROA-mean time course (red traces) of the back-projected V1 component. The VI components contributed to each peak in the ROA means, accounting for 44.7 and $73.0 \%$ of its variance in the two short-stimulus sessions (first and second rows of Fig. 3) and 79.5 and $83.5 \%$ in the two long-stimulus sessions (Figs. 2c and 3).

Figure 4 shows the component ROA maps and BOLD-image plots of a second category of components for the same subject those active in area MTN5 (BA 19). Again, although the active component maps were highly overlapping (POR $=87.4 \%$ ), the time courses as shown in the BOLD-image plots varied widely across trials. Components active in MTN5 were associated with complex and highly variable time courses not amenable to model using standard HR templates. Together, Figs. 2-4 show that HRs in different brain areas (e.g., V1 vs MTN5) may differ markedly across trials and stimulus parameters, even in the "simplest" event-related experimental paradigm.

The top panel of Fig. 5 shows ROAs of sample V1 components from the other five subjects. The upper and lower rows of BOLD-image plots show component activity in short-stimulus and long-stimulus sessions, respectively. The location of the active pericalcarine in region varied slightly between subjects. Possibly, this arose in part from the slightly different viewing angles for the different subjects. Note that the V1 component time courses varied widely between subjects, and within sessions, across trials. For clearer presentation, the BOLD-image plots in this figure were also smoothed with a vertical two-trial moving window and were color coded individually (see color bar scales).

\section{DISCUSSION}

We have shown that single-trial BOLD responses to infrequent presentations of flickering checkerboard stimuli may have time courses that vary systematically or unsystematically across stimulus parameters, visual brain areas, experimental sessions, and subjects. To visualize this variability, we introduced a new method of plotting event-related BOLD time courses, the BOLD image. BOLD-image plotting should also prove useful for plotting event-related BOLD time courses of single voxels or of arbitrary ROIs.

Infomax ICA decomposition was used to show that:

\section{Responsive Sensory Areas May Be Similar across}

\section{Sessions and Stimulus Parameters}

The brain locations of independent components active in V1 following stimulation, determined in separate ICA decompositions for each session, were highly replicable within subjects (Figs. 2-4) but differed somewhat between subjects, consistent with the report of Waldvogel et al. (2000) who used similar stimuli analyzed with correlational methods.

\section{Hemodynamic Responses to Simple Stimuli Areas May Be Surprisingly Variable Even in Primary Sensory Brain Areas}

Many fMRI studies have reported that changes in the subject performance strategy, habituation, learning, or aging all can produce large spatiotemporal differences in the BOLD signals recorded from the same subject performing the same task (D'Esposito et al., 1999; Williams et al., 2000). Expected hemodynamic responses may even be completely missing in some trials, reducing the statistical power of fixed models or templates (Miezin et al., 2000). Methods based on assumptions that HRs are reliably evoked by each stimulus, such as deconvolution approaches that identify HRs in BOLD data based on their temporal relationship to the stimulus presentation record, ignore the possibility that BOLD signals may not be the passive convolution of expected neural activity patterns with fixed HR kernels, plus Gaussian noise. Such models make questionable assumptions that the time course of brain metabolism can be predicted from the stimulus or event sequence, that the HR convolution process is static, and that noise is Gaussian.

ICA, on the other hand, makes no assumptions about either the shape of HRs or their consistency across trials. ICA does not rely on a convolutional model or assume that hemodynamic signals represent a fixed low-pass filter applied to neural activity levels. It is 
able to detect and separate functional brain areas with coherent BOLD time course features in common, even if they have omitted variable or multiple-peaked HRs. Moreover, ICA works well in non-Gaussian signals.

Applied to data from short-stimulus sessions, ICA returned V1-component HRs that included two major activity peaks in many trials. In these sessions, the first peak was more or less reliably timelocked to stimulus onsets, while the second peak varied in amplitude and latency across trials, appearing mostly in later trials. In long-stimulus sessions, the HR of the VI component contained, as initially expected, only a single peak, and this peak had larger amplitude and longer duration than the initial peak in the shortstimulus component HRs. It would have been difficult to extract these phenomena by searching through the data using a variety of expected or conceivable HR templates. The statistical power of such searches would be dissipated by their many degrees of freedom, making statistical confidence in the results low. We demonstrated, instead, a method of quantifying the contributions of independent components from regions of interest defined by component ROA.

Based on the results we present here, the sufficiency of the convolution model for analyzing sensory-driven $\mathrm{HRs}$, and the quality of the results derived from it, should be reconsidered. At least the actual time courses of identified active regions should be visualized using BOLD-image plots to check for response variability not a resembling constant noise background.

\section{Hemodynamic Responses to Visual Stimuli Can Vary Widely between Sensory Areas}

Our results (Figs. 4 and 5) show that the time courses of the V1-active and MTN5-active components differed substantially, even within the same subject and session. In area VI (Fig. 4), HRs with one and sometimes two major peaks were common. Time courses of MTN5 components (Fig. 5) were far less distinct and regular. This difference appears compatible with the hierarchic organization of the visual system. In the visual M-pathway, feed-forward information flows from V1 through V2 and MTN5 to the posterior parietal cortex. Since MTN5 integrates activity from other brain areas for motion detection (Zeki, 1993; Rees et al., 2000), its hemodynamics might be more context dependent than that in V1.

\section{Hemodynamic Responses Can Vary between}

Subjects, Even in Primary Sensory Areas

Comparison of the active component maps, BOLDimage plots, and averaged time courses for the V1active components in Fig. 5 reveals clear between-subject HR variability in spatial location and time course of the active regions. If it is necessary to combine results in V1 or other areas for different subjects, this variability could diffuse the resulting mean spatial map and time course, leading to Type I errors. Extra caution should thus be exercised during group analysis based on active map or time course averaging. It should also be expected that the quantity and quality of HR variability should increase in nonprimary sensory areas and particularly in frontal brain areas.

\section{Hemodynamic Responses Can Vary with Subject Strategy Changes and Attention Shifts}

Although we have no independent evidence to test such hypotheses on these data, our ICA results may have revealed unexpected changes in allocation of attention or task strategy of our subjects during the experiment. For example, although the flickering checkerboard stimuli we used sometimes triggered activation in MTN5, in the first five trials of the shortstimulus session of Subject 5 (Fig. 4, right column) such activation was small or absent. In later trials of the same session, the stimulus-evoked activation was more substantial. Response in these trials dominated the average time course (shown below the BOLD image). This difference might reflect top-down influences such as the subject paying increasing attention to the motion aspect of the stimulus in later trials. Top-down influences, including imagery, may contribute strongly to BOLD signal variability, although evidence to confirm these influences is limited (Huettle et al., 2001; Leonards et al., 2001).

\section{Usefulness of ICA for BOLD Signal Decomposition}

Conventional hypothesis-driven or model-based methods for analyzing fMRI data require a priori knowledge of the HR time courses and are not suitable for finding or measuring HRs with unknown or variable time courses. Variable HRs might be produced by several factors: by differences in the time course of blood drainage; by either deliberate or unwitting changes in subject performance strategy; by changes associated with learning or habituation; by variations in subject arousal, attention, or imagination; or by other unknown hemodynamic or artifactual processes. In such cases, ICA provides an effective data-driven method for separating the recorded BOLD signals into components accounting for activity of different brain or nonbrain processes, without relying on restrictive assumptions concerning the forms of the HR time courses or the spatial configurations of the affected brain areas.

We cannot expect, however, that ICA will always separate BOLD data into components that respect functional anatomic distinctions. As with any analysis method, the results of ICA can only be as meaningful as the goodness of fit between the method assumptions and the data. The main spatial assumption used by Infomax ICA-that small brain regions with mainly coherent BOLD time courses have substantially sepa- 
a

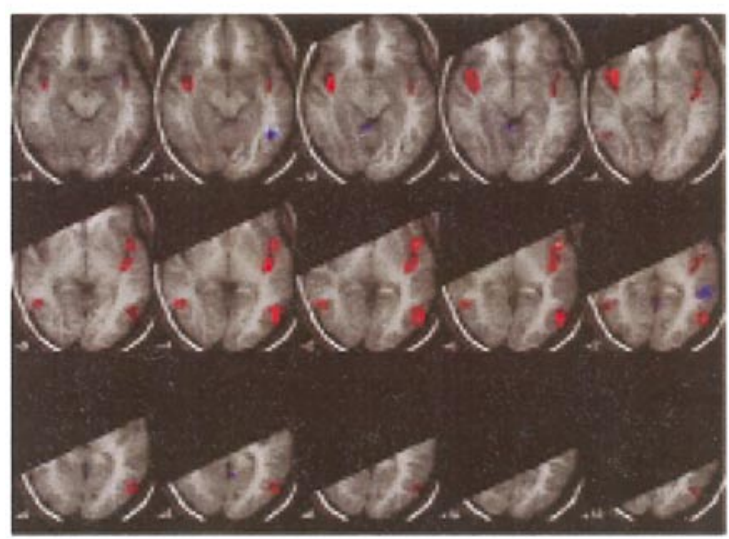

b

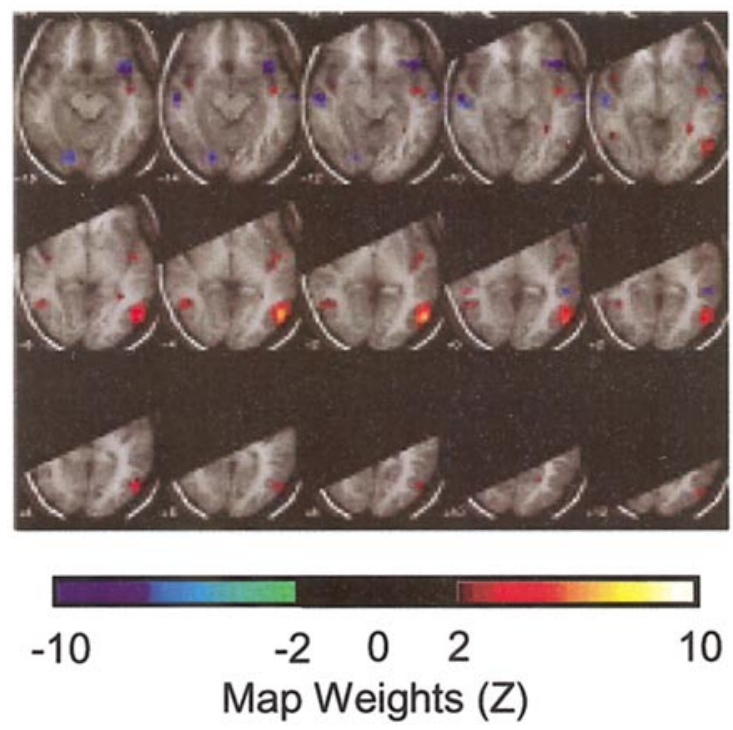

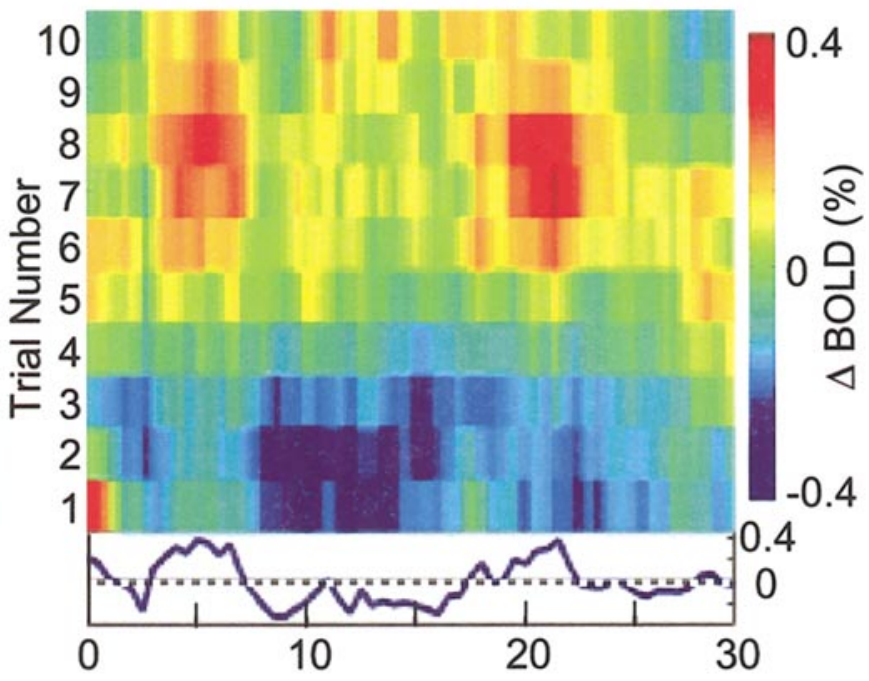

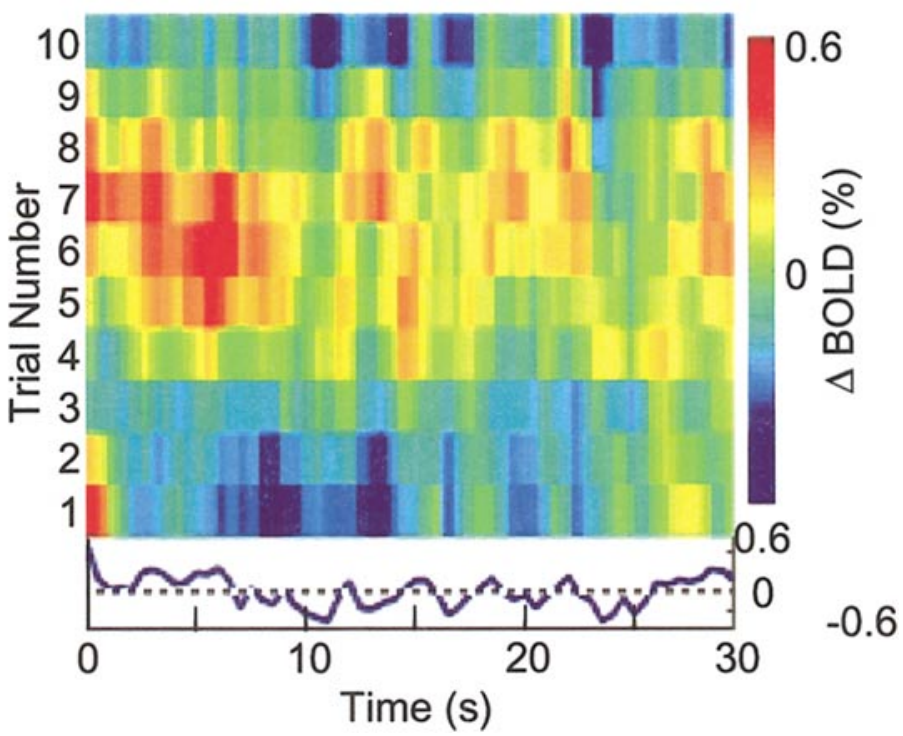

FIG. 4. Component ROA maps and BOLD-image plots of components active in area MTN5 for Subject 5 in (a) a short-stimulus session and (b) a long-stimulus session.

rate locations-appears physiologically reasonable and nonrestrictive. However, if the ROA of an active functional area changes across the training data, as with learning or exposure, ICA might separate its activity into separate consistently active and transiently active components with adjacent ROAs. Similar component splitting might occur if BOLD activity saturated in the most active voxels in a component ROA. Thus, best use of ICA for BOLD signal analysis requires visualization and comparison of ICA components, best accompanied by examination of convergent behavioral or other physiological evidence. The quantitative and visualization methods presented here should be helpful in this process. MATLAB tools for computing and visualizing results of ICA BOLD decomposition are available at http://sccn.ucsd.edu.

\section{CONCLUSIONS}

We have shown that brain HRs to sensory stimuli in an unstructured perception task may be trial-, site, stimulus-, and subject-dependent. In general, the assumption that a fixed combination of template functions can accurately map task-related brain areas and extract their time courses is questionable. Relying on template-based methods requires two leaps of faith. The first is that the temporal relationship between the task or stimulus sequence and brain metabolic activity is known in advance, allowing construction of a response template. The second is that the brain hemodynamic system acts as a static and therefore passive low-pass filter with a fixed or highly constrained impulse response. 


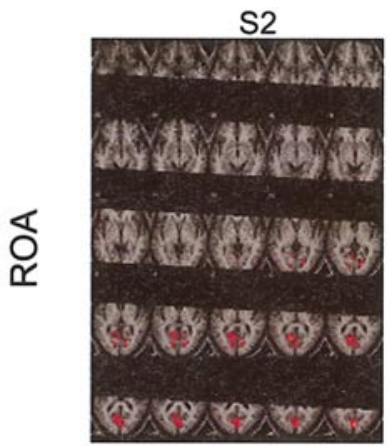

\#25

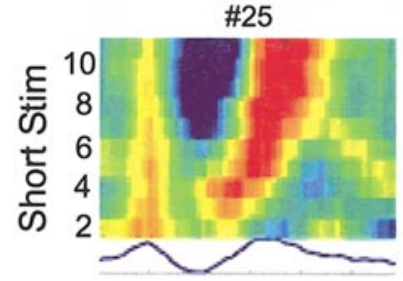

\#43

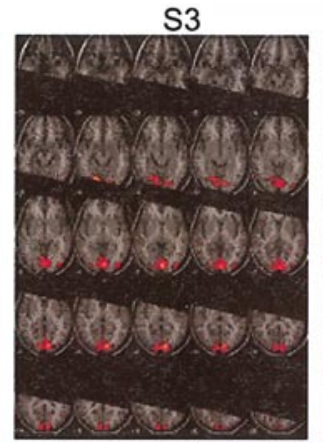

\#26

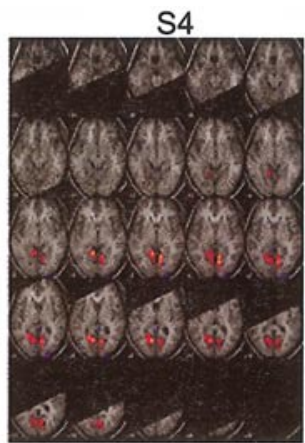

\#50

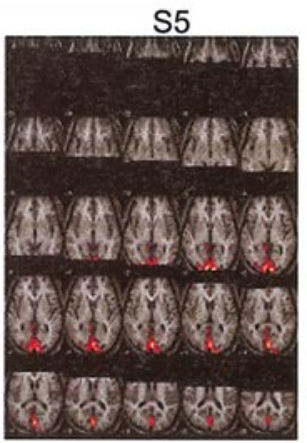

\#10
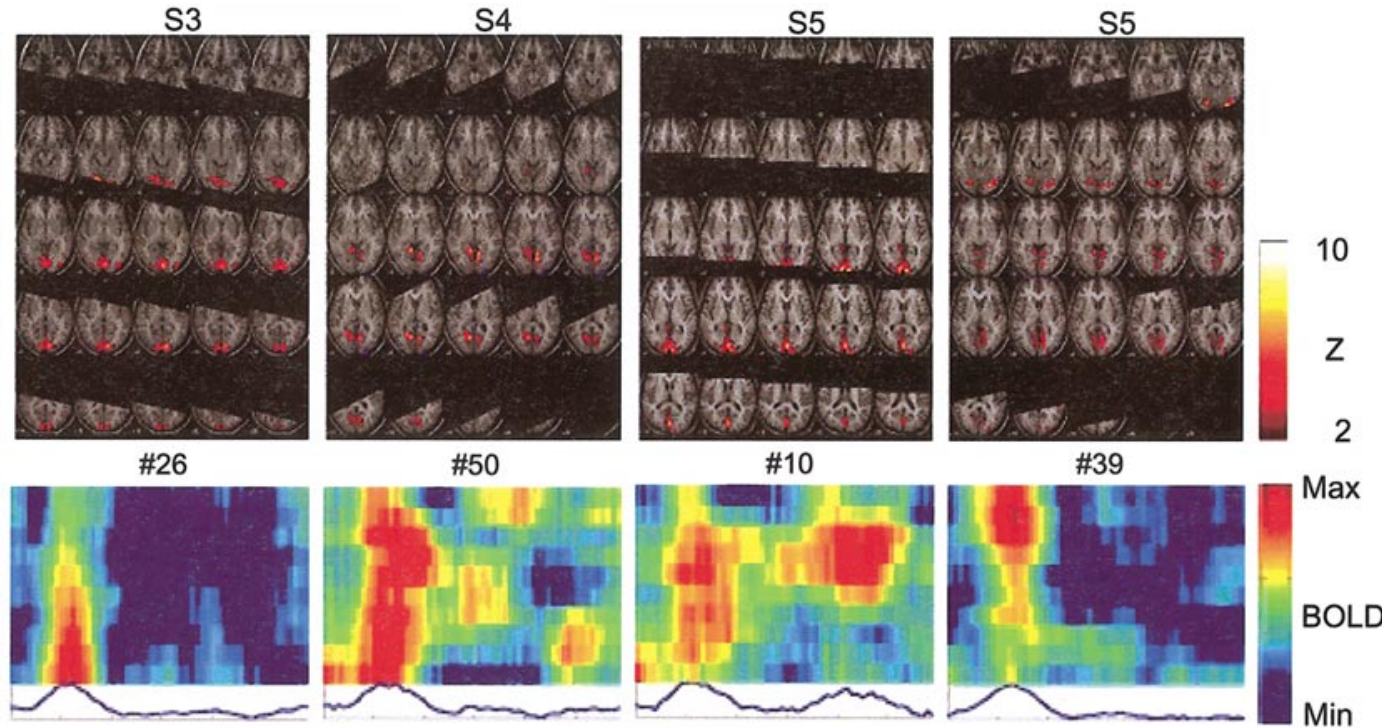

\#13

\#12

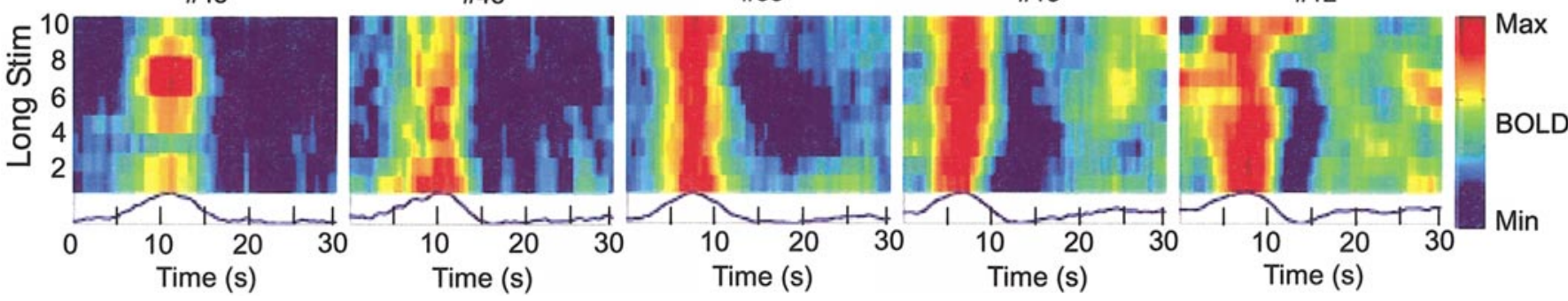

FIG. 5. BOLD-image plots from five other subjects. The upper block shows the VI component ROA map. The middle block displays the component time course in the short-stimulus session, and the lower block, the component time course in the long-stimulus session.

The use of ICA can complement hypothesis-driven methods for analyzing fMRI time series: (1) ICA does not rely on a priori knowledge of HR time courses and can be used to detect unforeseen, time-varying-, and site-dependent HRs; and (2) ICA can be used to separate the component processes corresponding to task-related metabolic responses, non-task-related physiological phenomena, and machine and movement artifacts (McKeown et al., 1998a,b; McKeown and Sejnowski, 1998).

Since ICA can identify a wide variety of time courses, it should expand the possible types of fMRI experiments that can be performed and meaningfully analyzed and interpreted. In particular, ICA might be able to reveal changes in the psychological state of subjects or patients. For example, it should aid in modeling BOLD changes tied to changes in subject performance, learning, or habituation. The exploration of nonstationary responses with ICA should allow fMRI research to consider the relationship between BOLD signal variability and shifting cognitive states that depend on motivation, arousal and intent. Although ICA reveals the complexity of BOLD signals, how best to interpret the resulting components is still unknown. Exploration of this question may play a key role in further development of functional brain imaging and analysis.

\section{ACKNOWLEDGMENTS}

This research was supported by the Swartz F oundation, the Office of Naval Research, the U.S. National Institutes of Health, the Howard Hughes Medical Institute, and the National Science Council of Taiwan and Ministry of Education of Taiwan (89BFA221401).

\section{REFERENCES}

Aguirre, G. K., Zarahn, E., and D'Esposito, M. 1998. The variability of human, BOLD hemodynamic responses. Neuroi maging 8:360369.

Andino, S. L. G., de Peralta Menendez, R. G., Thut, G., Spinelli, L., Blanke, O., Michel, C. M., Seeck, M., and Landis, T. 2000. Measuring the complexity of time series: an application to neurophysiological signals. Hum. Brain Map. 11:46-57

Arfanakis, K., Cordes, D., Haughton, V. M., Moritz, C. H., Quigley, M. A., and Meyerand, M. E. 2000. Combining independent component analysis and correlation analysis to probe interregional connectivity in $\mathrm{fMRI}$ task activation datasets. Magn. Reson. I maging 18:921-930.

Bandettini, P., J esmanowicz, A., Wong, E., and Hyde, J . 1993. Processing strategies for time-course data sets in functional MRI of human brain. Magn. Reson. Med. 30:161-173. 
Bandittini, P. A., Kwong, K. K., Davis, T. L., Tootell, R. B. H., Wong, E. C., Fox, P. T., Belliveau, J. W., Weisskoff, R. M., and Rosen, B. R. 1997. Characterization of cerebral blood oxygenation and flow changes during prolonged brain activation. Hum. Brain Map. 5:93-109.

Bell, A. J ., and Sejnowski, T. J . 1995. An information-maximization approach to blind separation and blind deconvolution. Neural Comput. 7:1129-1159.

Buckner, R. L. 1998. Event-related $\mathrm{fMRI}$ and the hemodynamic response. Hum. Brain Map. 6:373-377.

Buckner, R. L., Koutstaal, W., Schacter, D. L., Wagner, A. D., and Rosen, B. R. 1998a. Functional-anatomic study of episodic retrieval using fMRI. I. Retrieval effort versus retrieval success. Neurol mage 7:151-162.

Buckner, R. L., Koutstaal, W., Schacter, D. L., Dale, A. M., Rotte, M., and Rosen, B. R. 1998b. Functional-anatomic study of episodic retrieval. II. Selective averaging of event-related fMRI trials to test the retrieval success hypothesis. Neurol mage 7:163-175.

Calhoun, V. D., Adali, T., Pearlson, G. D., and Pekar, J . J . 2001. Spatial and temporal independent analysis of functional MRI data containing a pair of task-related waveforms. Hum. Brain Map. 13:43-53.

Clare, S., Humberstone, M., Hykin, J ., Blumhardt, L. D., Bowtell, R., and Morris P. 1999. Detection activations in event-related fMRI using analysis of variance. Magn. Reson. Med. 42:1117-1122.

Dale, A. M. 1999. Optimal experimental design for event-related fMRI. Hum. Brain Map. 8:109-114.

D'Esposito, M., Zarahn, E., Aguirre, G. K., and Rypma, B. 1999. The effect of normal aging on the coupling of neural activity to the bold hemodynamic response. Neurol mage 10:6-14.

Frahm, J ., Merboldt, K., Hanicke, W., Klenschmidt, A., and Boecker, H. 1994. Brain or vein oxygenation or flow? On signal physiology in functional MRI of human brain activation. NMR Biomed. 7:45-53.

Friston, K. J . 1995. Commentary and opinion. II. Statistical parametric mapping: Ontology and current issues. J . Cereb. Blood Flow Metab. 15:361-370.

Friston, K. J ., Ashburner, J ., Frith, C., Poline, J .-B., Heather, J ., and Frachowiak, R. 1995. Spatial registration and normalization of images. Hum. Brain Map. 2:165-189.

Friston, K. J . 1997. Imaging cognitive anatomy. Trend Cogn. Sci. 1:21-27.

Friston, K. J ., Fletcher, P., J osephs, O., Holmes, A., Rugg, M. D., and Turner, R. 1998. Event-related fMRI: Characterizing differential responses. Neurol mage 7:30-40.

Genovese, C. R., Noll, D. C., and Eddy, W. F. 1997. Estimating test-retest reliability in fMRI I. Mag. Reson. Med. 38:497-507.

Girolami, M. 1998. An alternative perspective on adaptive independent component analysis algorithms. Neural Comput. 10:2103-2114.

Gjedde, A. 1997. The relation between brain function and cerebral blood flow and metabolism. In Cerebrovascular Diseases. Lippincott-Raven, Philadel phia.

Hendrickson, A. E., and White, P. O. 1966. A method for the rotation of higher-order factors. Br. J . Math. Stat. Psychol. 19:97-103.

Huettel, S. A., Güzeldere, G., and McCarthy, G. 2001. Dissociating the neural mechanisms of visual attention in change detection using functional MRI. J . Cogn. Neurosci. 13:1006-1018.

J osephs, O., Turner, R., and Friston, K. 1997. Event-related fMRI. Hum. Brain Map. 5:243-248.

J ung, T.-P., Makeig, S., McKeown, M. J ., Bell, A. J ., Lee, T.-W., and Sejnowski, T. J . 2001a. I maging brain dynamics using independent component analysis. Proc. IEEE 89:1107-1122.

J ung, T.-P., Makeig, S., Westerfield, M., Townsend, J ., Courchesne, E., and Sejnowski, T. J . 2001b. Analysis and visualization of single-trial event-related potentials. Hum. Brain Map. 14:166-185.
Kansaku, K., Kitazawa, S., and Kawano, K. 1998. Sequential hemodynamic activation of motor areas and the draining veins during finger movements revealed by cross-correlation between signals from fMRI. NeuroReport 9:1969-1974.

Kruggel, F., and von Cramon, D. Y. 1999a. Modeling the hemodynamic response in single-trial functional MRI experiments. Magn. Reson. Med. 42:787-797.

Kruggel, F., and von Cramon, D. Y. 1999b. Temporal properties of the hemodynamic response in functional MRI. Hum. Brain Map. 8:259-271.

Kwong, K., Belliveau, J ., Chesler, D., Goldberg, I., Weisskoff, R., Poncelet, B., Kennedy, D., Hoppel, B., Cohen, M., Tunner, R., Cheng, H.-M., Brady, T., and Rosen, B. 1992. Dynamic magnetic resonance imaging. Proc. Natl. Acad. Sci. USA 89:5675-5679.

Lancaster, J . L., Woldorff, M. G., Parsons, L. M., Liotti, M., Freitas, C. S., Rainey, L., Kochunov, P. V., Nickerson, D., Mikiten, S. A., and Fox, P. T. 2000. Automated Talairach Atlas labels for functional brain mapping. Hum. Brain Map. 10:120-131.

Lee, A., Glover, G., and Mayer, G. 1995. Discrimination of large venous vessels in time-course spiral blood-oxygenation-dependent magnetic resonance function neuroimaging. Magn. Reson. Med. 33:745- 754.

Lee, T.-W., Girolami, M., and Sejnowski, T. J . 1999. Independent component analysis using an extended infomax algorithm for mixed sub-Gaussian and super-Gaussian sources. Neural Comput. 11:609- 633 .

Leonards, U., Sunaert, S., Van Hecke, P., and Orban, G. A. 2001. Attention mechanisms in visual search-An fMRI study. J . Cogn. Neurosci. 12:61-75.

Makeig, S. et al. 1998. MATLAB Tool box for I ndependent Component Analysis. http://www.cnl.salk.edu/ scott/ica.html.

Makeig, S., Westerfield, M., J ung, T.-P., Covington, J ., Townsend, J ., Sejnowski, T. J ., and Courchesne, E. 2000. Functionally independent components of the late positive event-related potential during visual spatial attention. J . Neurosci. 19:2665-2680.

McKeown, M. J ., J ung, T.-P., Makeig, S., Brown, G. G., Lee, T.-W., Kindermann, S. S., and Sejnowski, T. J . 1998a. Spatially independent activity patterns in functional MRI data during the Stroop color-naming task. Proc. Natl. Acad. Sci. USA 95:803- 810.

McKeown, M. J ., Makeig, S., Brown, G. G., J ung, T.-P., Kindermann, S. S., Bell, A. J ., and Sejnowski, T. J . 1998b. Analysis of fMRI data by blind separation into independent spatial components. Hum. Brain Map. 6:160-188.

McKeown, M. J ., and Sejnowski, T. J . 1998. Independent component analysis of $\mathrm{fMRI}$ data: Examining the assumptions. Hum. Brain Map. 6:368-372.

Mckeown, M. J . 2000. Detection of consistently task-related activations in fMRI data with hybrid independent component analysis. Neurol mage 11:24-35.

Menon, R. S., Luknowsky, D. C., and Gati, J . S. 1998. Mental chronometry using latency-resolved functional MRI. Proc. Natl. Acad. Sci. USA 95:10902-10907.

Miezin, F. M., Maccotta, L., Ollinger, J. M., Peterson, S. E., and Buckner, R. L. 2000. Characterizing the hemodynamic response: Effects of presentation rate, sampling procedure, and the possibility of ordering brain activity based on relative timing. Neurol mage 11:735-759.

Moeller, J ., and Strother, S. 1991. A regional covariance approach to the analysis of functional pattern in positron emission tomography data. J . Cereb. Blood Flow Metab. 11:A121-A135.

Moritz, C. H., Haughton, V. M., Cordes, D., Quigley, M., and Meyerand, M. E. 2000. Whole-brain functional MR imaging activation from a finger-tapping task examined with independent component analysis. Am. J. Neuroradiol. 21:1629-1635.

Ogawa, S., Tank, D., Menon, R., Ellermann, J ., Kim, S., Merkle, H., 
and Ugurbil, K. 1992. Intrinsic signal changes accompanying sensory stimulation: Functional brain mapping with magnetic resonance imaging. Proc. Natl. Acad. Sci. USA 89:5951-5959.

Rajapakse, J . C., Kruggel, F., Maisog, J . M., and von Cramon, D. Y. 1998. Modeling hemodynamic response for analysis of functional MRI time-series. Hum. Brain Map. 6:283-300.

Rees, G., Friston, K., and Koch, C. 2000. A direct quantitative relationship between the functional properties of human and macaque V5. Nature Neurosci. 3:716-723.

Rowe, B. D. 2001. Bayesian source separation for reference function determination in fMRI. Mag. Reson. Med. 46:374-378.
Waldvogel, D., van Gelderen, P., Immisch, I., Pfeiffer, C., and Hallett, M. 2000. The variability of serial fMRI data: Correlation between a visual and a motor task. NeuroReport 11:3843-3847.

Williams L. M., Brammer, M. J ., Skerrett, D., Lagopolous, J ., Rennie, C., Kozek, K., Olivieri, G., Peduto, T., and Gordon, E. 2000. The neural correlation of orienting: An integration of $\mathrm{FMRI}$ and skin conductance orienting. NeuroReport 11:3011-3015.

Zarahn, E., Aguirre, G., and D’Esposito, M. 1997. A trial-based experimental design of fMRI. Neurol mage 6:122-138.

Zeki, S. 1993: Chapter 20: The $P$ and M pathways and the 'what and where' doctrine. In A Vision of the Brain. Blackwell, London. 